\title{
The impact of newborn screening on cystic fibrosis testing in Victoria, Australia
}

\author{
M E Balnaves, L Bonacquisto, I Francis, J Glazner, S Forrest
}

\begin{abstract}
Newborn screening for cystic fibrosis (CF) by examining the levels of immunoreactive trypsinogen was introduced in Victoria in 1989. This was modified by the addition of testing for the common CF gene mutation, $\Delta F 508$, in 1990. Problems with the first newborn screening protocol were overcome with the addition of the DNA test as there was no need to contact the majority of families, there was a reduced number of sweat tests, and less anxiety was experienced by parents. The mode of diagnosis changed from failure to thrive, steatorrhoea, rectal prolapse, and family history to diagnosis through newborn screening. Newborn screening dramatically reduced the time of diagnosis of CF to approximately six weeks or less in the majority of cases. Since the introduction of newborn screening, the uptake of prenatal diagnosis in CF families has increased two and a quarter fold.
\end{abstract}

(F Med Genet 1995;32:537-542)

Newborn screening for phenylketonuria was first performed in 1962 by Guthrie. ${ }^{1}$ It started in Victoria in 1966 on a voluntary basis, reaching full population coverage by 1971 . Screening for congenital hypothyroidism by thyrotrophin assay was added in $1976,{ }^{2}$ and for cystic fibrosis (CF) by immunoreactive trypsinogen (IRT) in $1989 .^{3}$ The newborn screening programme is now administered by the Victorian Clinical Genetics Service (VCGS) as a fully funded government programme. Dried blood spot screening samples are collected routinely (parents are given the opportunity to refuse participation in the programme) in all maternity centres from 48 hours of age. New screening tests are added to the programme as the result of consultation and agreement between the relevant medical specialist group, the VCGS, and the Victorian Government. In the case of CF screening, the decision process made use of the data from the New South Wales programme which started in $1981 .^{4}$

With respect to CF testing, it was shown that infants with raised IRTs in the first few months of life were more likely to have CF than those with normal results. ${ }^{3}$ Infants whose IRT remained raised after a repeat test had their sweat electrolytes analysed to determine their CF status as this was regarded as the definitive test for CF. ${ }^{5}$ However, IRT levels could also be influenced by a number of other factors, including respiratory infections, gut abnormal- ities, or premature birth ${ }^{6}$ and hence a large number of false positives were detected by this system.

The gene that is mutated in patients with $\mathrm{CF}$, the cystic fibrosis transmembrane conductance regulator (CFTR), was discovered in September of $1989 .{ }^{7} \mathrm{~A} 3 \mathrm{bp}$ deletion in the genomic DNA resulting in the removal of the amino acid phenylalanine at position 508 in the protein $(\Delta F 508)$ was shown to be the most common mutation causing CF. The $\Delta \mathrm{F} 508$ mutation accounts for approximately $70 \%$ of CF gene mutations in our population. This varies depending on the ethnic group (CF consortium). In July 1990 , the DNA test for $\Delta F 508$, which can be performed directly on dried blood spot samples, ${ }^{89}$ was made part of the newborn screening programme in Victoria.

This paper presents the Victorian newborn screening results for CF up to 1993 as well as specifically tracking the process of identification of subjects with CF over a two year time span (1991-1992). The uptake of prenatal diagnosis by the families identified through newborn screening is also examined and is shown to increase in comparison to families where the CF affected child was diagnosed before the advent of newborn screening.

\section{Methods and materials}

Primers flanking the $3 \mathrm{bp}$ deletion, $\Delta \mathrm{F} 508,{ }^{7}$ amplify a $98 \mathrm{bp}$ fragment in normal people while a $95 \mathrm{bp}$ fragment is present in people with the deletion. The dried blood spot samples are eluted in a mixture of water, standard $1 \times$ buffer $(6.7 \mathrm{mmol} / 1$ Tris- $\mathrm{HCl} \mathrm{pH} 8.8$, $1.66 \mathrm{mmol} / \mathrm{l}\left(\mathrm{NH}_{4}\right)_{2} \mathrm{SO}_{4}, 0.045 \%$ Triton X$100,20 \mu \mathrm{g} / \mathrm{ml}$ gelatin), $2 \mathrm{mmol} / 1 \mathrm{MgCl}_{2}$ and $0.1 \%$ Triton $\mathrm{X}-100$ at $37^{\circ} \mathrm{C}$ for 30 minutes. Samples are boiled for five minutes and placed on ice briefly. A mixture of dNTPs (final concentration $0.2 \mathrm{mmol} / \mathrm{l}), 500 \mathrm{ng}$ each primer, and 2.5 units Taq polymerase is added in a final $100 \mu \mathrm{l}$ reaction and overlaid with paraffin oil. The PCR is performed for 27 cycles for one minute at $95^{\circ} \mathrm{C}$, one minute at $63^{\circ} \mathrm{C}$, and two minutes at $72^{\circ} \mathrm{C}$ in a thermal cycler. A $20 \mu \mathrm{l}$ aliquot of the PCR product is run on a $10 \%$ non-denaturing polyacrylamide gel (NOVEX, Australia) and visualised by ethidium bromide staining. The two fragments are easily resolved.

Sample data were analysed from the newborn screening laboratory for the last four years. Data before 1990, when DNA testing began, were obtained from personal records of patients seen for CF testing in the genetics clinic of the Royal Children's Hospital. This was cross referenced with data on all patients seen by the 
Table 1 Comparing the mode of diagnosis of CF in Victoria from 1987 to 1993

\begin{tabular}{|c|c|c|c|c|c|c|c|c|}
\hline Year & 1987 & 1988 & $\begin{array}{l}\text { IRT start } \\
1989\end{array}$ & $\begin{array}{l}\text { DNAt start } \\
1990\end{array}$ & 1991 & 1992 & $\begin{array}{l}1993 \\
(30.9 .93)\end{array}$ & Total \\
\hline No of infants tested & 61221 & 63247 & 63826 & 66719 & 64762 & 65946 & 48620 & - \\
\hline No of CF diagnosed & 26 & 29 & 29 & 22 & 26 & 24 & 11 & 167 \\
\hline Chest infection & 3 & 3 & 0 & 1 & 1 & 0 & 0 & 8 \\
\hline Symptoms & 10 & 14 & 2 & $i$ & 0 & 1 & 1 & 29 \\
\hline Meconium ileus* & 3 & 3 & 3 & 4 & 4 & 5 & 2 & 24 \\
\hline Affected sib & 5 & 4 & 1 & 0 & 0 & 2 & 0 & 12 \\
\hline Other & 3 & 1 & 1 & 0 & 0 & 0 & 0 & 5 \\
\hline Newborn screening & 0 & 0 & 17 & 13 & 20 & 15 & 7 & 72 \\
\hline Chest + symptoms & 1 & 2 & 2 & 0 & 1 & 1 & 0 & 7 \\
\hline Mode not given & 1 & 2 & 3 & 3 & 0 & 0 & 1 & 10 \\
\hline
\end{tabular}

All years are potentially incomplete as children with mild features may not yet have presented clinically with $\mathrm{CF}$ * Samples counted independently from newborn screening.

tDNA testing performed only on a subset of infants.

Department of Thoracic Medicine at the Royal Children's Hospital since 1987.

In determining the uptake of prenatal diagnosis, 1992 results were not included. Couples were arbitrarily given a two and a half year time period in which to reproduce after the birth of their first affected child. Families who took longer than two and a half years to have a child, whether they used prenatal diagnosis or not, were not included. The data were not analysed beyond 1991, as these families would not have had the full two and a half year time interval in which to reproduce.

Results

Table 1 represents the mode of diagnosis of CF from 1987 to the end of September 1993. Before 1989, failure to thrive, steatorrhoea, rectal prolapse (included under symptoms in table 1), and a sib affected with CF were the main modes of diagnosis for CF. In 1989, when the IRT test was introduced, newborn screening became the main mode of diagnosis and has remained so. Infants with meconium ileus (MI) are flagged before entry into newborn screening and were counted as a separate category in terms of mode of diagnosis. Data may not be complete since the total number of infants with CF could be higher for all these years, as those with mild features may not yet have presented clinically.

Fig 1 compares the original newborn screening protocol for $\mathrm{CF}$ with the new improved protocol incorporating DNA analysis. The top $0.7 \%$ of infants with raised IRT levels were followed up in each protocol. However, in the original protocol a repeat blood sample was required while in the new protocol, DNA testing for the $\Delta \mathrm{F} 508$ mutation can be performed

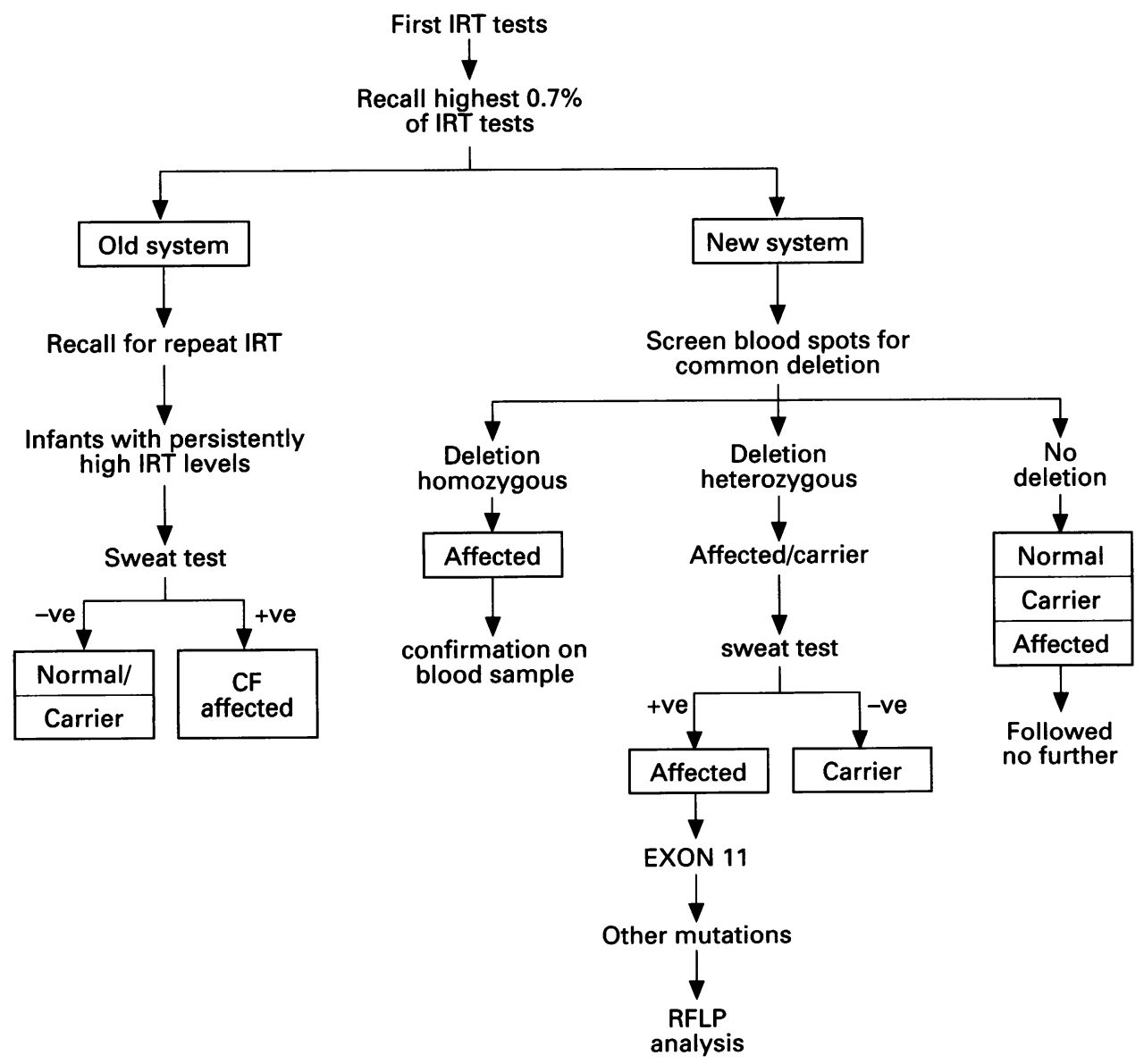

Figure 1 Comparison of the old and new screening protocols with the introduction of $4 F 508$ testing. 

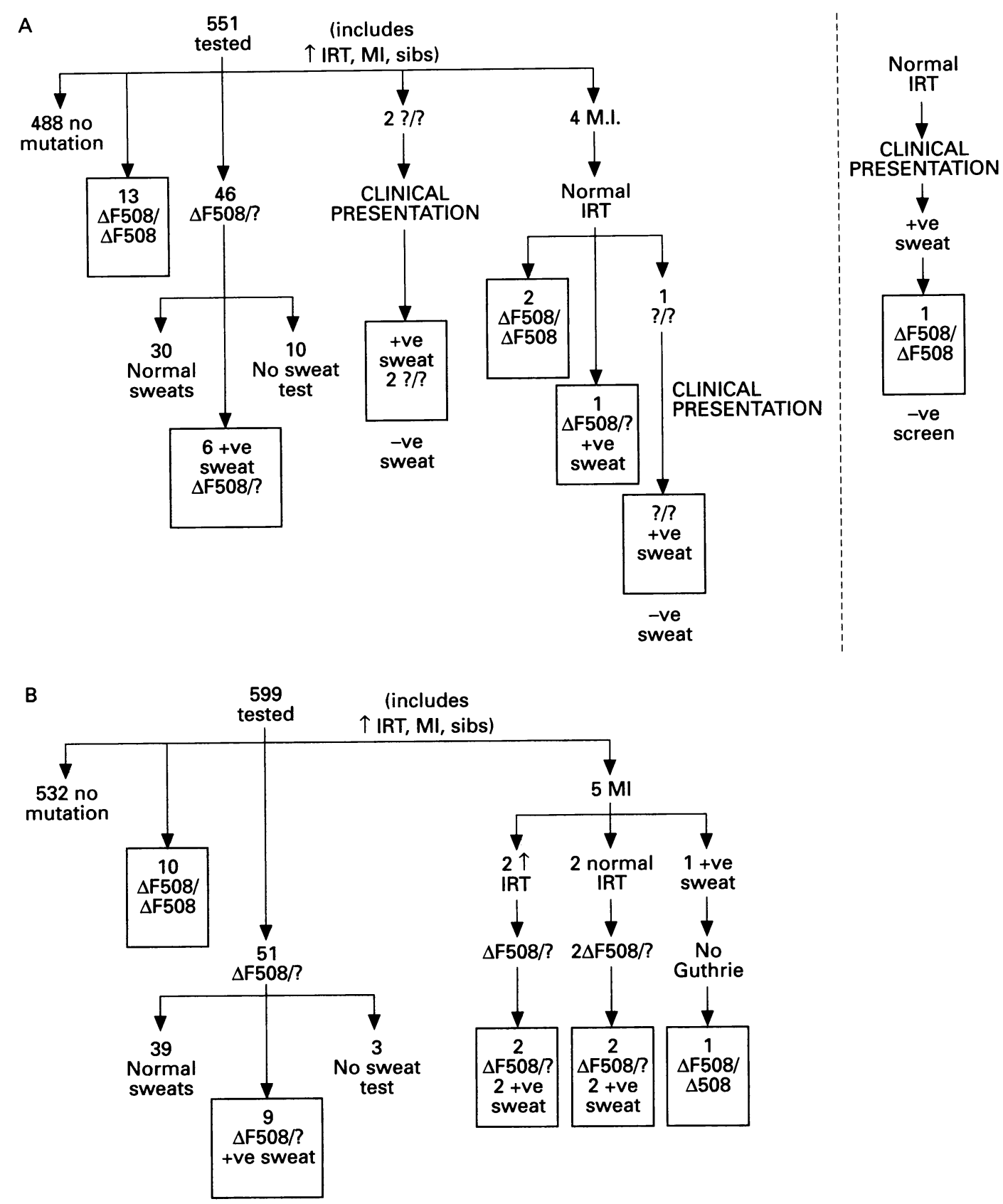

Figure 2 Diagnosis of CF patients in (A) 1991 and (B) 1992.

on the original dried blood spot sample so a second sample is not required. The advantage of not having a repeat IRT test is that it reduces the number of patients recalled and the anxiety experienced by parents. Infants homozygous for $\Delta$ F508 are clearly affected and a confirmatory blood sample is requested to remove the possibility of sample mix up. Infants heterozygous for the $\Delta \mathrm{F} 508$ mutation have a sweat test performed to determine their CF status as they can either be carriers or may be affected with $C F$ but have an as yet unidentified $C F$ gene mutation on the other chromosome. Those with positive sweat test results have further DNA testing performed (exon 11 mutations, other CF mutations, and RFLP analysis, if necessary). Infants who do not have the mutation on either chromosome are not followed any further.

Data on the identification of CF affected infants born in 1991 and 1992 were used to trace the path of these infants through the modified newborn screening protocol (fig $2 \mathrm{~A}$, B). In 1991, 551 infants were tested for $\Delta F 508$. These included infants with a raised IRT and others that were prompted into the system such as those suffering from $\mathrm{MI}$ and sibs of $\mathrm{CF}$ patients. Meconium ileus can result in a lowered IRT. These infants are often not represented in the top $0.7 \%$ of IRT levels. As most of the infants with $\mathrm{MI}$ actually have $C \mathrm{~F}^{10}$ it is important to identify samples from these infants so that they are not missed in the system and can proceed straight to DNA testing.

Of the 551 infants tested, 488 had no mutation and were followed no further, 13 were homozygous $\Delta F 508 / \Delta F 508$, and 46 were heterozygous. Of these 46,30 had normal sweat test results, six had positive sweat test results and hence were affected with CF (and went on to have further testing as outlined in fig 1 ), and 10 had no sweat test in Melbourne. Of 

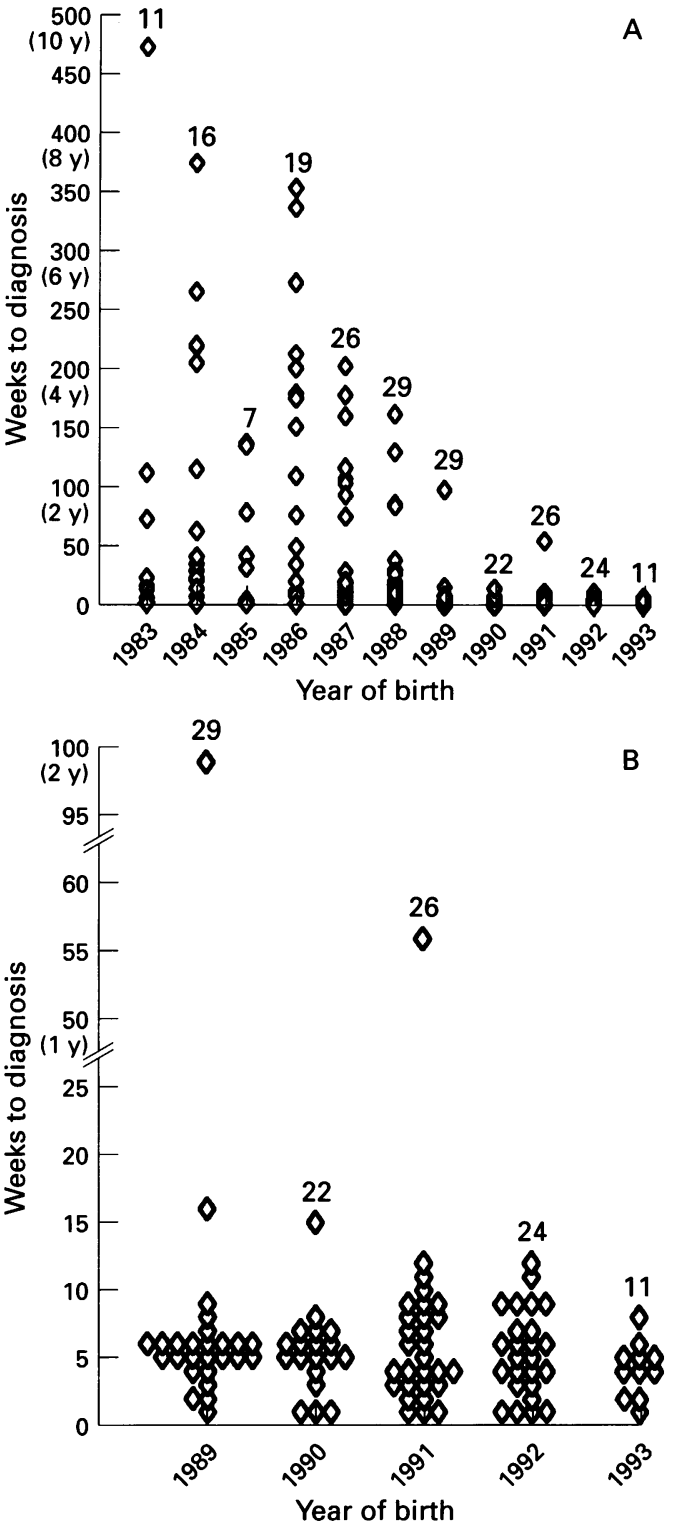

Figure 3 Age at diagnosis of CF. (A) From 1983 to 1993. The total number of $C F$ patients is indicated at the top of each year. Numbers above each year do not indicate the total number of $C F$ children born each year but only the children where information was available for the time taken to diagnose CF. (B) Expanded view from 1989 to 1993 since the introduction of newborn screening.

these 10, two died, two were sweat tested elsewhere, two declined, one had a family history of CF, one was lost to the system, and two had mothers with CF. Four infants were identified with $M I$ and despite normal IRTs were tested for $\Delta \mathrm{F} 508$. Two were homozygous, one was heterozygous and had a positive sweat test, and one had no $\Delta$ F508 mutation. The infant with no $\Delta F 508$ mutation later presented clinically and had a positive sweat test. In 1991, four infants were missed through newborn screening. Two of these four had raised IRT levels but no $\Delta F 508$ mutations, presented clinically, and were then confirmed by a sweat test. One infant had a normal IRT, presented clinically, had a positive sweat test, and was subsequently shown to be homozygous for $\Delta$ F508. The last infant had MI, a normal IRT, no $\Delta F 508$ mutation, and later presented clinically and was confirmed by a sweat test.
In 1992, 599 infants were tested for $\Delta \mathrm{F} 508$; 532 had no mutation and were followed no further (fig 2B). Ten were homozygous $\Delta \mathrm{F} 508$ and 51 were $\Delta F 508$ heterozygotes. Of these heterozygotes, 39 had normal sweat tests, nine were positive (and went on to have further testing as outlined in fig 1 ), and three had no sweat test. Of these one had died, one infant had a mother with CF, and one had a sib with CF. There is no indication why they were followed no further. Again five infants were identified with MI and all were shown to be affected. No misses have been diagnosed yet in infants born in 1992, but infants with mild features may not yet have presented clinically.

Since the advent of newborn screening for CF in 1989, we have diagnosed 112 infants affected with CF. Fifty four were $\Delta F 508$ homozygotes and 39 were affected heterozygotes (DNA results for 1989 were determined retrospectively). An additional five infants with $\mathrm{CF}$ did not have $\Delta F 508$ on either chromosome and were diagnosed clinically.

A study of age at diagnosis of CF shows that this has been dramatically reduced since the advent of newborn screening, as can be seen in the graph (fig 3A). The age at diagnosis before 1989 varied from 1 to 10 years. In general, this time is now approximately six weeks, when the sweat tests are performed in $\Delta F 508$ heterozygous infants. Infants homozygous for $\Delta \mathrm{F} 508$ are diagnosed even earlier. Even after the introduction of newborn screening, there are the occasional few who take longer to diagnose, 10 to 12 weeks or 1 to 2 years (fig 3B). This is because some infants have extremely mild phenotypes and do not carry the common mutations for which our laboratory tests.

In order to determine if newborn screening had made an impact on the uptake of prenatal diagnosis of CF, data were analysed from 1986 to 1991 for families using prenatal diagnosis for CF. We have full ascertainment of women having prenatal diagnosis for cystic fibrosis in Victoria over this time interval as we are the only laboratory in Victoria performing this service. The years 1986-1988 were the years before newborn screening and 1989-1991 were the years after screening was introduced. Families with more than one affected child had these children without prenatal diagnosis and hence were considered as "no CVS" if the birth fell into the two and a half year time interval. The number of $\mathrm{CF}$ affected children listed for each year represent the birth of the index case in the family and do not include subsequent $\mathrm{CF}$ affected children in the same family. The data are shown in table 2 .

Table 2 Usage of prenatal diagnosis between 1986 and 1991

\begin{tabular}{lll}
\hline & $\begin{array}{l}\text { CVS } \leq 2 y \\
\text { mth }\end{array}$ & $\begin{array}{l}\text { Year proband in CF family born: } \\
\text { total CF }\end{array}$ \\
\hline 1986 & 2 & 20 \\
1987 & 1 & 23 \\
1988 & 4 & 27 \\
1989 & 6 & 27 \\
1990 & 4 & 20 \\
1991 & 6 & 24 \\
\hline
\end{tabular}




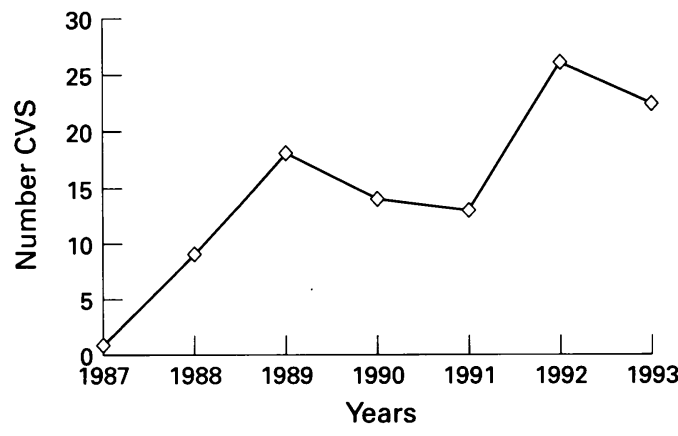

Figure 4 Number of prenatal diagnoses for $C F$ since 1987.

Using the program Epilnfo, the $\chi^{2}$ value was calculated to compare the usage of prenatal diagnosis for 1986-1988 with 1989-1991. From 1986-1988, 7/70 families (10\%) used prenatal diagnosis. From 1989-1991, 16/71 families $(22 \cdot 5 \%)$ used prenatal diagnosis. Therefore, there was a two and a quarter fold increase in the uptake of prenatal diagnosis (uncorrected $\chi^{2}=4.06$ and $\mathrm{p}=0.04,95 \% \mathrm{CI}$ $0 \cdot 99-5 \cdot 14)$, that is, the increased likelihood that a woman would have a CVS for CF in 1989-1991 compared to women in the 19861988 period is of borderline significance. These findings support the hypothesis that with the introduction of newborn screening, the uptake of prenatal diagnosis will be higher than it has been in the past.

Preliminary analysis of the results shows that 14 of the 72 families identified since the advent of newborn screening have already used prenatal diagnosis for a future pregnancy, three of them twice and one family three times. Seven families are known to have had a second child without using prenatal diagnosis and one who would have but for a miscarriage. CVS usage has been recorded for Victorian CF families from 1987 until 1993. Results are shown in fig 4. The number of chorionic villus samples performed each year has generally increased and it is anticipated that there will be an increase in the uptake of prenatal diagnosis in the future as more time elapses from the birth of the first affected child. Since 1987, 102 prenatal diagnoses have been performed for $\mathrm{CF}$ and 29 were normal, 41 were carriers, and 32 were affected.

As part of our quality control, all CVS results are followed up. When infants are born, dried blood spot samples are obtained and the informative test is repeated for all carriers and normal subjects. Fetal material is always requested from terminations. Of the $32 \mathrm{CVS}$ samples with affected results, 31 were terminated. Products of conception were received from 13, which were all confirmed. In total, $84 / 102$ were followed up with $100 \%$ accuracy.

Requests for carrier testing have become commonplace since the discovery of the $\Delta \mathrm{F} 508$ mutation, its relatively high frequency in the population, and the ease of performing the test. Many of the people requesting carrier testing are relatives of the infants recently diagnosed by newborn screening. Carrier testing is only performed in families with known CF affected subjects or in families where the infant was found to be heterozygous for $\Delta \mathrm{F} 508$ on newborn screening but had a negative sweat test result. Three hundred and fifty six people (to 30.9.93) have been tested for $\Delta F 508$ and three exon 11 mutations: G542X, G551D, and R553X. This has identified three carrier couples, two who have since used prenatal diagnosis and one who has as yet not used prenatal diagnosis. In another couple, the partner was shown to be a carrier while the sib of the affected child had his risk reduced to zero as he had not inherited the CF mutation in the family.

\section{Discussion}

Newborn screening for cystic fibrosis was introduced into Victoria in $1989 .{ }^{3}$ The screening test comprised an immunoreactive trypsinogen (IRT) assay on the newborn dried blood spot sample, followed by another IRT on a 28 day sample for those whose initial IRT fell within the top $0.7 \%$. The discovery of the CF gene in $1989^{7}$ enabled direct mutation testing to be incorporated into the screening assay. In the new scheme, which was introduced in 1991, the second IRT assay was replaced by an assay for the most common CF mutation, $\Delta F 508$ (70\% of CF mutations). This rapid, simple, and accurate assay can be performed directly on the newborn dried blood spot sample ${ }^{89}$ and has been incorporated in other newborn screening programmes. ${ }^{11}$ A separate DNA extraction step is not required and the need for a second sample collection is eliminated. Testing for mutations other than $\Delta \mathrm{F} 508$ has not been included in our screening programme. Analysis of our CF data shows that the next most common mutation, G551D ( $5 \%$ of CF mutations), was found in only seven cases. However, all were compound heterozygotes for $\Delta \mathrm{F} 508$ and so would be detected by the current protocol.

Farrell and Mischler ${ }^{12}$ identified some areas of concern with regard to newborn screening for CF. In their hands, the IRT test had suboptimal sensitivity and inadequate positive predictive value, thereby leading to adverse effects, particularly in the false positive families. They noted that the discovery of the CF gene would possibly solve some of these problems. They concluded that the costs of screening did not significantly add to the costs of care for CF patients, but after only five years of randomised investigation, it was too soon to reach conclusions on whether newborn screening conferred any benefits in terms of reduced admissions to hospital and better prognosis.

Newborn screening for CF was criticised by Gitzelmann ${ }^{13}$ because it does not fit the accepted model of a treatable disorder. Unlike treatment for phenylketonuria etc, where early medical intervention prevents most of the manifestations of the disease, treatment for $\mathrm{CF}$ only delays the onset of symptoms and the eventual outcome. In addition, he stated that the screening protocol in its original form suffered from a high false positive rate and would have little effect on reproductive decisions. Tluczek et $a l^{1415}$ also discussed the 
adverse effect of false positives in the Wisconsin screening programme with particular emphasis on increased parental anxiety.

We feel that some of these criticisms have been met by the new screening protocol we have adopted. The high false positive rate in the original protocol resulted from the need to obtain a second sample from infants in the top $0.7 \%$ of IRT results. This has been eliminated by performing mutation analysis on the first dried blood spot sample. This has reduced the need to contact parents, which in the past caused much of the parental anxiety, from $0.7 \%$ down to $0.08 \%$ of infants tested. Only those infants found to be heterozygous for $\Delta \mathrm{F} 508$ require a confirmatory sweat test, since homozygosity for $\Delta \mathrm{F} 508$ is, itself, confirmatory of CF. This knowledge is also of great assistance in reducing parental anxiety by appropriate counselling, in contrast to the original screening protocol in which the indication for a second sample or a sweat test was the non-specific rise of IRT.

We have shown that the newborn screening test for CF has dramatically reduced the age at diagnosis (fig 3A, B), thus providing timely treatment of early symptoms. In a five year study in Wales and the West Midlands ${ }^{16}$ with IRT measurement taken on infants born in alternate weeks, the mean age at diagnosis was significantly lower in the screened group in comparison to the clinically diagnosed group. In addition, the screened group spent a significantly shorter time in hospital in the first year of life.

Wilcken et $a l^{4}$ have shown that reduction in age at diagnosis has some major benefits. Before screening, they found that the delay between onset of symptoms and diagnosis of CF was on average $2 \cdot 6$ years. During this period, parents suffered from uncertainty and anxiety and, despite symptoms being present in almost all infants from the age of 3 months, appropriate treatment was inevitably delayed, thus impacting adversely on the infants' prognosis. In contrast, after the introduction of newborn screening, the average delay until diagnosis was six weeks, thus permitting prompt treatment.

A study by Helton $e t a^{17}$ of parental attitudes to newborn screening for CF supports the benefits of early diagnosis. The parents favoured newborn screening because the infants did not suffer the misdiagnoses and admissions to hospital of the unscreened group. There has been some evidence in published reports that early diagnosis improves outcome. ${ }^{418}$

The early diagnosis of cystic fibrosis as a result of newborn screening allows parents the option of genetic counselling before subsequent pregnancies and prenatal diagnosis is a practical possibility. ${ }^{19}$ Our figures show an increase in prenatal diagnosis for CF since the introduction of newborn screening. Unlike the experience of others, ${ }^{17}$ all but one of our families using prenatal diagnosis have opted to terminate affected pregnancies. In our experience, the reasons for couples not making use of prenatal diagnosis were mainly religious or lack of understanding of the nature of the disease and the availability of the test. This indicates the need for better public education on genetic diseases and newborn screening. In contrast, in families with longer standing cases of $\mathrm{CF}$, the increased awareness of the nature of $\mathrm{CF}$ within these families has generated requests from relatives for carrier testing, with the view towards prenatal diagnosis if both members of a couple are found to be carriers.

\section{Conclusions}

The impact of newborn screening for cystic fibrosis has been widespread. Families have benefited from the early diagnosis that newborn screening allows. For the affected child, prompt treatment of symptoms is possible although the effect on long term prognosis is yet to be determined. For the parents of the affected child, informed decisions about their reproductive future can be made sooner. Extended family members are now also aware of their risk of being CF carriers and can be counselled appropriately.

We thank Jane Halliday for her help with the $\chi^{2}$ analysis.

1 Guthrie R. The origin of newborn screening. Screening 1992; 1:5-15.

2 Francis I. Newborn screening in Australia and New Zealand 1984-1990. Med $\mathcal{F}$ Aust 1991;155:821-3.

3 Crossley J, Elliot R, Smith P. Dried-blood spot screening for cystic fibrosis in the newborn. Lancet 1979;i:472-4. 4 Wilcken B, Towns SJ, Mellis CM. Diagnostic delay in cystic
fibrosis: lessons from newborn screening. Arch Dis Child 1983;58:863-6.

5 Gibson LE, Cooke RE. A test for concentration of electrolytes in sweat in cystic fibrosis of the pancreas utilizing pilocarpine by iontophoresis. Pediatrics 1959;23:545-9.

6 Ravine D, Francis RI, Danks DM. Non-specific elevation of immunoreactive trypsinogen in sick infants. Eur $₹$ Pediatr 1993;152:348-9.

7 Kerem B-S, Rommens JM, Buchanan JA, et al. Identification of the cystic fibrosis gene: genetic analysis. Science 1989; 245:1073-80.

8 Schwartz EI, Khalchitsky SE, Eisensmith RC, Woo SLC Polymerase chain reaction amplification from dried blood spots on Guthrie cards. Lancet 1990;336:639-40

9 Nelson PV, Carey WF, Morris CP. Gene amplification directly from Guthrie blood spots. Lancet 1990;336:14512.

10 Rusakow LS, Abman SH, Sokol RJ, et al. Immunoreactive trypsinogen levels in infants with cystic fibrosis complicated by meconium ileus. Screening 1993;2:13-17.

11 Ranieri E, Ryall RG, Morris CP, et al. Neonatal screening strategy for cystic fibrosis using immunoreactive trypsinogen and direct gene analysis. $B M F$ 1991;302:1237-40.

12 Farrell PM, Mischler EH. Newborn screening for cystic fibrosis. The Cystic Fibrosis Neonatal Screening Study Group. Adv Pediatr 1992;39:35-70.

13 Gitzelmann R. Why we should not screen our newborns for cystic fibrosis. Helv Paediatr Acta 1981;36:493-4.

14 Tluczek A, Mischler EH, Farrell PM, et al. Parents' knowledge of neonatal screening and response to false-positive cystic fibrosis testing. Dev Behav Paediatr 1992;13:181-6.

15 Tluczek A, Mischler EH, Bowers B, et al. Psychological impact of false positive results when screening for cystic fibrosis. Paediatr Pulmonol Suppl 1991;7:29-37.

16 Chatfield S, Owen G, Ryley HC, et al. Neonatal screening for cystic fibrosis in Wales and the West Midlands: clinica assessment after five years of screening. Arch Dis Child 1991;66:29-33.

17 Helton JL, Harmon RJ, Robinson N, Accurso FJ. Parental attitudes toward newborn screening for cystic fibrosis. Paediatr Pulmonol 1991;S7:23-8.

18 Orenstein DM, Boat TF, Stern RC, et al. The effect of early diagnosis and treatment of cystic fibrosis; a seven yea study of 16 sibling pairs. $A m \mathcal{F} D$ is Child $1977 ; 131: 973-5$.

19 Green MR, Weaver LT, Heeley AF, et al. Cystic fibrosis identified by neonatal screening: incidence, genotype, and earty natural history. Arch Dis Child 1993;68:464-7. 